DrAft VERSION NOVEMBER 8, 2018

Preprint typeset using $\mathrm{LAT}_{\mathrm{E}} \mathrm{X}$ style emulateapj v. 5/2/11

\title{
DISCOVERY OF A REDBACK MILLISECOND PULSAR CANDIDATE: 3FGL J0212.1+5320
}

\author{
Kwan-Lok Li ${ }^{1}$, Albert K. H. Kong ${ }^{2}$, Xian Hou ${ }^{2,3,4}$, Jirong Mao ${ }^{3,4}$, Jay Strader ${ }^{1}$, Laura Chomiuk ${ }^{1}$, Evangelia \\ TREMOU $^{1}$ \\ Draft version November 8, 2018
}

\begin{abstract}
We present a multi-wavelength study of the unidentified Fermi object, 3FGL J0212.1+5320. Within the 95\% error ellipse, Chandra detects a bright X-ray source (i.e., $\left.F_{0.5-7 \mathrm{keV}}=1.4 \times 10^{-12} \mathrm{erg} \mathrm{cm}^{-2} \mathrm{~s}^{-1}\right)$, which has a low-mass optical counterpart $\left(M \lesssim 0.4 M_{\odot}\right.$ and $T \sim 6000 \mathrm{~K})$. A clear ellipsoidal modulation is shown in optical/infrared at 20.87 hours. The gamma-ray properties of 3FGL J0212.1+5320 are all consistent with that of a millisecond pulsar, suggesting that it is a $\gamma$-ray redback millisecond pulsar binary with a low-mass companion filling $\gtrsim 64 \%$ of the Roche-lobe. If confirmed, it will be a redback binary with one of the longest orbital periods known. Spectroscopic data taken in 2015 from the Lijiang observatory show no evidence of strong emission lines, revealing that the accretion is currently inactive (the rotation-powered pulsar state). This is consistent with the low X-ray luminosities $\left(L_{X} \approx 10^{32} \mathrm{erg} \mathrm{s}^{-1}\right)$ and the possible X-ray modulation seen by Chandra and Swift. Considering that the X-ray luminosity and the high X-ray-to- $\gamma$-ray flux ratio $(8 \%)$ are both comparable to that of the two known $\gamma$-ray transitional millisecond pulsars, we suspect that 3FGL J0212.1+5320 could be a potential target to search for future transition to the accretion active state.
\end{abstract}

Subject headings: binaries: close — gamma rays: stars — pulsars: general — X-rays: binaries

\section{INTRODUCTION}

Progenitors of millisecond pulsars (MSPs), though not yet fully understood, are believed to be neutron stars in low-mass X-ray binaries (LMXBs). According to the recycling scenario (Alpar et al. 1982), the neutron stars are spun up through accretion from the late-type companions (if any) to ultimately evolve into MSPs. Through the so-called LMXB Case A channel (Tauris 2011), a compact binary (i.e., orbital period $<1$ day) consisting of a MSP and a very low-mass companion (which was striped by the neutron star and/or partially "evaporated" by the energetic pulsar wind/ $\gamma$-rays; Chen et al. 2013) remains at the very end phase of such an evolution, known as black widow (BW; companion mass: $<0.1 M_{\odot}$ ) or redback (RB; companion mass: $\sim 0.1-0.4 M_{\odot}$ ) binaries. A few RBs, known as transitional MSPs, have already shown remarkable transition(s) between the LMXB state and the radio pulsar state in optical, X-rays, and/or $\gamma$ rays (i.e., M28I; Papitto et al. 2013, PSR J1023+0038; Archibald et al. 2009; Patruno et al. 2014, and PSR J1227-4853; Roy et al. 2015), clearly indicating the close relationship between LMXBs and radio MSPs. $\mathrm{BW} / \mathrm{RBs}$ are interesting objects, not to mention the fascinating theoretical interpretation of multi-wavelength observations for individual studies (e.g., the keV-to-GeV emission models of PSR J1023+0038 in different states; Li et al. 2014; Papitto \& Torres 2015). They also pro-

\footnotetext{
${ }^{1}$ Department of Physics and Astronomy, Michigan State University, East Lansing, MI 48824, USA; liliray@pa.msu.edu(KLL)

2 Institute of Astronomy and Department of Physics, National Tsing Hua University, Hsinchu 30013, Taiwan

${ }^{3}$ Yunnan Observatories, Chinese Academy of Sciences, Kunming, 650216, China

${ }^{4}$ Key laboratory for the Structure and Evolution of Celestial Objects, Chinese Academy of Sciences, Kunming, 650216, China
}

vide crucial information on the long-term accretion history. In particular, BWs are the key to uncover how the companions are finally eliminated, after which isolated MSPs are formed van den Heuvel \& van Paradijs 1988).

As MSPs are powerful $\gamma$-ray sources with strong $\mathrm{GeV}$ magnetospheric radiations (e.g., from the outer gap, the slot gap, or the polar cap; Cheng et al. 1986; Muslimov \& Harding 2003; Ruderman \& Sutherland 1975) and/or the inverse-Compton $\gamma$-ray emissions of the pulsar wind nebulae when the accretion is active (Takata et al. 2014; Li et al. 2014), many of them should have been detected by Fermi-LAT as a class of unidentified Fermi object (UFO), the second-largest population detected by Fermi-LAT (Acero et al. 2015). Although not all the UFOs are MSPs (in fact many of them are thought to be AGNs, the largest source class in the catalog), good BW/RBs candidates can be selected based on the $\gamma$-ray spectral curvatures and the $\gamma$-ray variabilities (Rav et al. 2012; Kong et al. 2012, 2014; Hui et al. 2015b) and confirmed their pulsar natures by detecting the radio/ $\gamma$-ray pulsations. Thanks to the Fermi Pulsar Search Consortium (PSC), a great success has been achieved in discovering new pulsars through "blind" searches for coherent pulsations in radio and $\gamma$-rays (Ray et al. 2012), and the known BW and RB populations have been greatly extended in recent years.

Alternatively, multi-wavelength studies of UFOs are the secondary way to search for $\mathrm{BW} / \mathrm{RBs}$ MSP candidates. In most of the cases, X-ray follow-ups are the key to narrow down the source location, allowing identification of the optical counterparts. Once the optical counterpart is identified, time-series optical observations can test the $\mathrm{BW} / \mathrm{RB}$ identity by searching for the orbital modulation on timescale of 
hours produced by pulsar irradiation on the companion and/or ellipsoidal variation. Through this multiwavelength technique, several UFOs, for examples, 2FGL J1311.7-3429/PSR J1311-3430 (Pletsch et al. 2012), 1FGL J1417.7-4407/PSR J1417-4402 (not a canonical BW/RB system; Strader et al. 2015; (Camilo et al.|2016), and 1FGL J2339.7-0531/PSR J2339-0533 (Kong et al. 2012; Pletsch \& Clark 2015) have been identified as MSP binaries and some of them have been confirmed by the detection of millisecond radio/ $\gamma$-ray pulsations, proving the validity of the method.

In this paper, we report the discovery of a $\gamma$-rayemitting RB candidate, 3FGL J0212.1+5320. In the following sections, we present multi-wavelength studies using the optical imaging/spectroscopic data from the Lijiang (Fan et al. 2015), Lulin, and Michigan State University (MSU) observatories, the Chandra X-ray data, and the Fermi-LAT third source catalog (3FGL; Acero et al. 2015). Discussions will be given in the last section.

\section{THE GAMMA-RAY PROPERTIES IN 3FGL}

3FGL J0212.1+5320 is an unidentified bright $\gamma$-ray source (i.e., $F_{\gamma}=(1.71 \pm 0.16) \times 10^{-11} \mathrm{erg} \mathrm{cm}^{-2} \mathrm{~s}^{-1}$ in $0.1-100 \mathrm{GeV}$, which is top $15 \%$ among the sources in 3FGL; Acero et al. 2015) that was first detected by Fermi-LAT in $\gamma$-rays in the Fermi-LAT first source catalog (1FGL; Abdo et al. 2010). It also later appears in 3FGL with a detection significance of $25 \sigma$.

Based on the second Fermi-LAT pulsar catalog (Abdo et al. 2013), the $\gamma$-ray properties of pulsars can be characterized by a low source variability and a curved $\gamma$-ray spectral shape. Although they are not necessary conditions, 3FGL J0212.1+5320 fulfils both of the criteria (Table 10, suggesting its possible pulsar nature in $\gamma$-rays. Similar to many other $\gamma$-ray pulsars that have seen stable in $\gamma$-rays over years Abdo et al. 2010), 3FGL J0212.1+5320 can also be considered as a steady source with a small 3FGL variability index of 51.47 (i.e., for a source with a variability index larger than 72.44 , there is a less than $1 \%$ chance of being a steady source; Acero et al. 2015). In addition, the $\gamma$ ray spectrum of 3FGL J0212.1+5320 is probably more than a single power-law but rather with an extra curvature component (e.g., an exponential cut-off) as the spectral curve significance is $6.3 \sigma$ in $3 \mathrm{FGL}$, which is also another common feature among the pulsars detected in 3FGL (Acero et al. 2015). In fact, Saz Parkinson et al. (2016) and Mirabal et al. (2016) have found that 3FGL J0212.1+5320 is a strong MSP candidate, using statistical and machine learning techniques.

\section{SWIFT AND CHANDRA X-RAY OBSERVATIONS}

As one of the survey targets in the Swift/XRT survey of Fermi unassociated sources (Stroh \& Falcone 2013), 3FGL J0212.1+5320 has been observed twice by Swift/XRT in October 2010 (the observations are separated by 3 days with a total exposure time of $4.5 \mathrm{ks})$. Within the 95\% 3FGL error ellipse, a bright $\mathrm{X}$-ray counterpart was detected and listed as 1SXPS J021210.6+532136 in the Swift/XRT point source catalog (1SXPS; Evans et al. 2014). According to 1SXPS, the source is located at $\alpha(\mathrm{J} 2000)=02^{\mathrm{h}} 12^{\mathrm{m}} 10.62$, $\delta(\mathrm{J} 2000)=+53^{\circ} 21^{\prime} 36^{\prime \prime} .8$ (90\% positional uncertainty: $\left.3.8^{\prime \prime}\right)$ with a mean count rate of $(2.26 \pm 0.26) \times 10^{-2} \mathrm{ct} \mathrm{s}^{-1}$. A moderate flux variability is seen between the two observations from $(2.61 \pm 0.32) \times 10^{-2}$ ct s$^{-1}$ to $(1.31 \pm 0.41) \times$ $10^{-2}$ ct s $^{-1}$ in 3 days (equivalent to a $2.9 \sigma$ change). The X-ray spectrum could be described by an absorbed power-law of $N_{\mathrm{H}}=1.4_{-1.4}^{+2.8} \times 10^{21} \mathrm{~cm}^{-2}$ (the Galactic column density $N_{\mathrm{H}}=1.5 \times 10^{21} \mathrm{~cm}^{-2}$; Kalberla et al. 2005) and $\Gamma_{X}=1.0_{-0.4}^{+0.5}$ with an unabsorbed flux of $F_{0.3-10 \mathrm{keV}}=1.6_{-0.3}^{+0.5} \times 10^{-12} \mathrm{erg} \mathrm{cm}^{-2} \mathrm{~s}^{-1}(W$-stat $=$ 57.98 and $\chi^{2}=63.02$; dof $=78$ ). Alternatively, the spectrum could be fitted with an APEC thermal plasma model, however, with an extremely high and poorly constrained plasma temperature (i.e., $k T \sim 100 \mathrm{keV}$ ). As the best-fit temperature is just too high to be physical, we do not further consider the APEC model in the following analyses.

Chandra has also observed the field of view once with ACIS for $30 \mathrm{ks}$ in 2013 August (Obs ID: 14814; PI: Saz Parkinson) and 1SXPS J021210.6+532136 is clearly detected at $\alpha(\mathrm{J} 2000)=02^{\mathrm{h}} 12^{\mathrm{m}} 10 \mathrm{~s} 50, \delta(\mathrm{J} 2000)=$ $+53^{\circ} 21^{\prime} 38^{\prime \prime} .9$ (90\% positional uncertainty: $\left.0.8^{\prime \prime}\right)$ with a net count rate of $(9.03 \pm 0.17) \times 10^{-2}$ ct s$^{-1}(0.5$ $7 \mathrm{keV})$. With a total number of 2685 photon counts, we binned the data to have at least 20 counts per bin and fitted the binned spectrum with an absorbed power-law. The best-fit parameters are $N_{\mathrm{H}}=(1.4 \pm$ $0.5) \times 10^{21} \mathrm{~cm}^{-2}, \Gamma_{X}=1.3 \pm 0.1$, and $F_{0.5-7 \mathrm{keV}}=$ $(1.35 \pm 0.06) \times 10^{-12} \mathrm{erg} \mathrm{cm}^{-2} \mathrm{~s}^{-1}$ (or $F_{0.3-10 \mathrm{keV}}=(1.89 \pm$ $0.08) \times 10^{-12} \mathrm{erg} \mathrm{cm}^{-2} \mathrm{~s}^{-1} ; \chi^{2}=98.78$ and $\left.d o f=105\right)$, which are all consistent with that extracted from the Swift/XRT data and the Chandra spectral fitting by Saz Parkinson et al. (2016). To examine the short term variability seen by Swift/XRT, we extracted a 4000-sec bin lightcurve with the Chandra/ACIS data and a flux variability on an hourly timescale is clearly shown (Figure 11). To quantify the variability significance, we computed the $\chi^{2}$ value of the 8 data bins with a flat lightcurve model, which is $\chi^{2}=24.39($ of $f=7)$, indicating that there is only a $0.1 \%$ chance that the variability is produced by random fluctuation.

\section{OPTICAL DATA}

At the Chandra X-ray position, we found a bright optical counterpart $(R=14.23 \mathrm{mag})$ in the USNO-B1.0 cata$\log$ (Monet et al. 2003), USNO-B1.0 1433-0078846, with an offset of $0.2^{\prime \prime}$. The same source is also detected in the 2MASS (Skrutskie et al. 2006) and WISE (Wright et al. 2010) catalogs. Using the multi-epoch photometry table of WISE5, a variability of $0.2-0.3$ mag is clearly seen in the $w 1$ band data of 33 epochs taken in 2010 February and August. The modulation is likely periodic with a period of 10-20 hours (see Figure 15 for the modulation, although the phase light curve was folded at 20.87 hours).

\subsection{Imaging from the $M S U$ and Lulin Observatories}

A monitoring campaign with the $0.6-\mathrm{m}$ telescope in the MSU observatory and the 1-m telescope in the Lulin observatory has been carried out from 2015 October to 2016 January to investigate the $\sim 10-20 \mathrm{~h}$ modulation seen in

\footnotetext{
5 http://irsa.ipac.caltech.edu/Missions/wise.html
} 

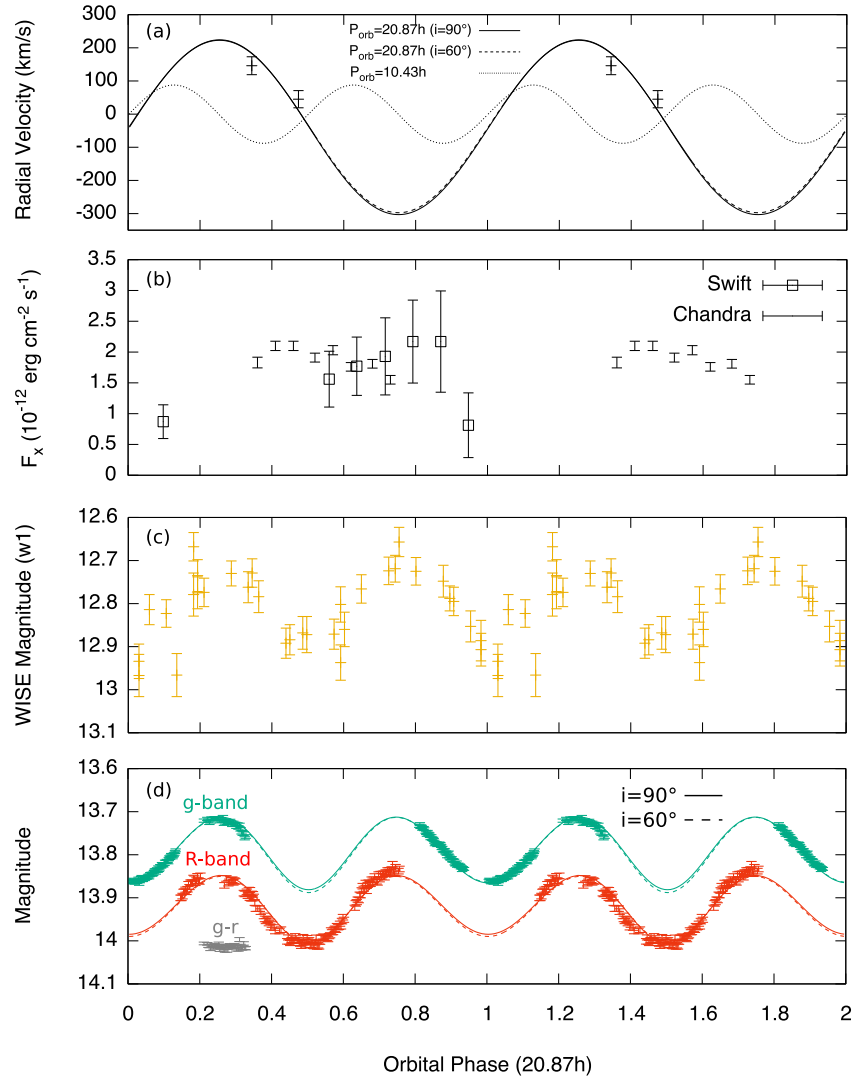

FIG. 1.- The plots show several physical quantities versus orbital phase $\left(P_{\text {orb }}=20.8698 \mathrm{~h}\right)$, including $(\mathrm{a})$ the radial velocities with the ELC models at $20.87 \mathrm{~h}$ (solid line for $i=90^{\circ}$ and dashed line for $i=60^{\circ}$ ) and a $10.43 \mathrm{~h}$ model curve (with an arbitrary amplitude; dotted line) projected on the $20.87 \mathrm{~h}$ orbital phase for comparison, (b) the X-ray flux (the Swift data is only shown in the first cycle for a clear view of the Chandra data in the second cycle), (c) the WISE $w 1$-band data, and (d) the $g$ - and $R$-band data tentatively calibrated with the UCAC4 Catalog (Zacharias et al. 2013) and the extinction of $A_{v}=0.4992 \mathrm{mag}$ (Schlafly \& Finkbeiner 2011) with the ELC models and the $(g-r)$ with an arbitrary offset. Two cycles are shown for clarity.

WISE. We observed the source for 3 consecutive nights from October 10 to October 12 with the $0.6-\mathrm{m}$ telescope in the $R$-band (200/300 sec for each frame, depending on the weather) and with the 1-m telescope in the SDSS $r$ and $g$-bands for 3 other nights (i.e., November 8/9 and January 9 ; only $g$-band images were taken on the first two nights and both $r$ - and $g$-band were taken by turns on the last night; $60 / 120 \mathrm{sec}$ for the $r / g$-band images, respectively).

\subsection{Spectroscopy from the Lijiang Observatory}

Two 1200-sec medium-resolution optical spectra (5750-8800 $\AA$ ) were taken on 2015 November 4 and 5 with the 2.4-m telescope at the Lijiang observatory. After (i) the standard reduction processes with the IRAF package ONEDSPEC, (ii) a flux calibration with the standard star $\mathrm{BD}+28^{\circ} 4211$ (Oke 1990), and (iii) an extinction correction with $A_{v}=0.4992$ mag Schlafly \& Finkbeiner 2011; which is roughly consistent with the $N_{\mathrm{H}}$ value estimated by Chandra) and the Cardelli extinction law (Cardelli et al. 1989), the calibrated data show spectral shapes comparable to that of a low-mass star (Figure 2) without any accretion features. After matching the data with the synthetic spectra from the Munari online library6 (Munari et al. 2005; a solar metallicity of $[\mathrm{M} / \mathrm{H}]=0$ and a typical $\mathrm{RB}$ rotational broadening of $V=100 \mathrm{~km} \mathrm{~s}^{-1}$ are assumed), we found that the spectra can be best described by $T=5750 \mathrm{~K}$ and $\log g=4.5$ (Figure 2), of which the stellar properties are very close to the $M \approx 0.4 M_{\odot}$ low-mass companion of the RB PSR J2129-0429 (Bellm et al. 2016). Therefore, we tentatively assume the secondary star of 3FGL J0212.1+5320 to be around $M \sim 0.4 M_{\odot}$.

\section{DETAILED TIMING ANALYSES \\ 5.1. Orbital Period Determination}

After applying the standard data reduction procedures by IRAF on the optical imaging data and removing some bad frames due to bad tracking or bad weather, we used a differential photometry technique to study the optical modulation, which shows a clear sinusoidal shape in all bands (Figure 11). We fitted all the data (including the WISE data; all are heliocentric corrected) simultaneously with sinusoidal functions with common period and phases, but different amplitudes and baselines for each data set. The best-fit period is 10.43479(7) hours (corresponding to the pulsar irradiation case) or 20.8698(1) hours (the ellipsoidal variation case) with the flux minimum epoch at HJD 2457305.5551(4) (the phase zero of Figure 1 and the following timing analyses). It is worth noting that the data used span over 5 years of time (i.e., from 2010 to 2015), which leads to a very high accuracy of the best-fit period. The best-fit amplitudes of the bands are roughly consistent with each other within a largest offset of $0.02 \mathrm{mag}$ (i.e., $a_{w 1}=0.09 \pm 0.02 \mathrm{mag}$, $a_{R}=0.0845 \pm 0.0009 \mathrm{mag}, a_{g}=0.0731 \pm 0.0004 \mathrm{mag}$, and $\left.a_{r}=0.092 \pm 0.007\right)$. In particular, the simultaneous $r$ - and $g$-band data taken by Lulin on January 9 do not show any clear color evolving trend during the phase interval of $\phi_{10}=0.42-0.66$ at $P=10.43 \mathrm{~h}$ (or $\phi_{20}=0.21-0.33$ at $P=20.87 \mathrm{~h}$; Figure 1 d), suggesting that there is likely no strong orbital color variability. This indicates that the pulsar irradiation effect on the companion is very limited and thus the modulation is probably caused by ellipsoidal variation.

\subsection{Radial Velocity Measurement}

Following the method described in Bellm et al. (2016), we first removed the telluric lines of the Lijiang spectra by omitting bands of $6860-7000 \AA, 7570-7700 \AA, 7150-$ $7350 \AA$, and $8100-8400 \AA$. Using the RVSAO Package of IRAF, we used the task xcsao to calculate the barycentric corrected radial velocities by cross-correlating the spectral data with the $T=5750 \mathrm{~K}$ synthetic spectrum (all the spectra involved are automatically normalized during the cross-correlation process). Both the spectra were found to be red-shifted with radial velocities of $136 \pm 19 \mathrm{~km} \mathrm{~s}^{-1}$ (Nov 4) and $31 \pm 17 \mathrm{~km} \mathrm{~s}^{-1}$ (Nov 5). By applying the $20.87 \mathrm{~h}$ (or 10.43h) ephemeris, the orbital phases of the radial velocities are $\phi_{20}=0.34$ (or $\left.\phi_{10}=0.69\right)$ and $\phi_{20}=0.47$ (or $\phi_{10}=0.95$ ), respectively. For the pulsar irradiation case (i.e., the orbital period is 10.43 hours), the companion should be moving from the behind of the pulsar to the front in the orbital interval

6 http://archives.pd.astro.it/2500-10500/ 


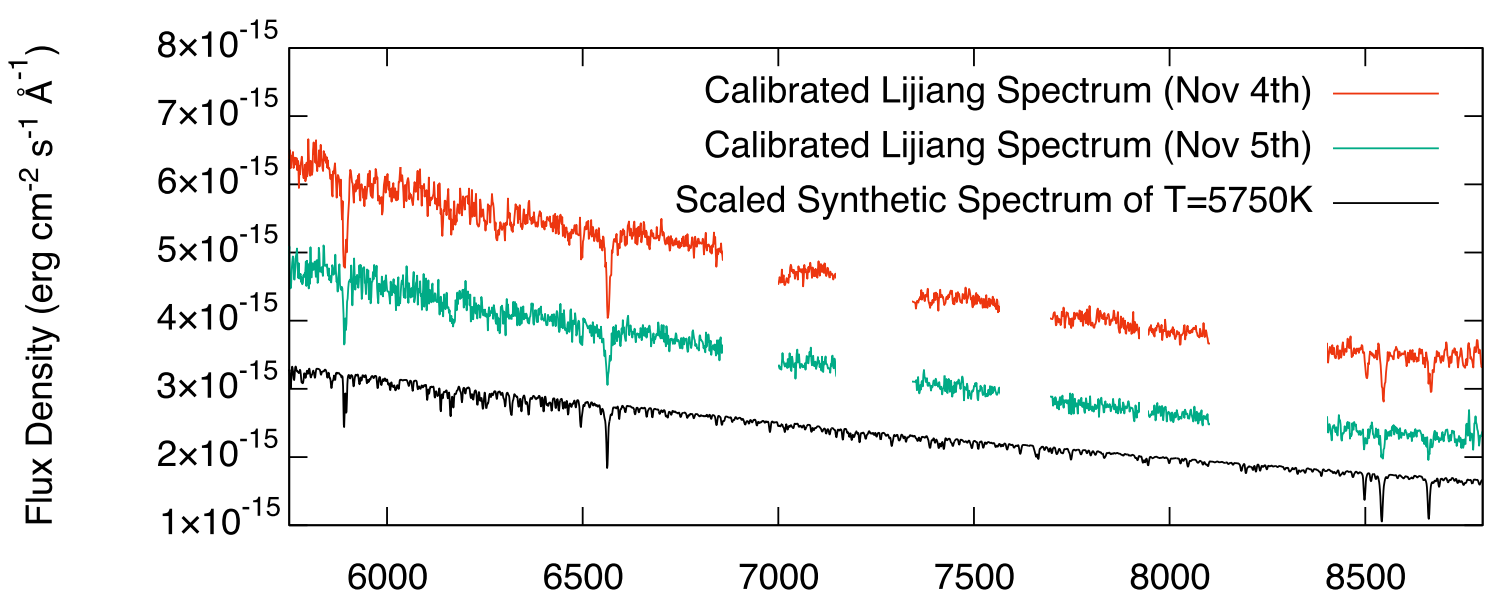

Wavelength (Angstrom)

FIG. 2.- From top to bottom, the curves are the two Lijiang spectra of USNO-B1.0 1433-0078846 taken on November 4th and 5th, and the model spectrum of $T=5750 \mathrm{~K}$ from the Munari synthetic spectral library. There are four gaps present on the Lijiang spectra due to the removal of the telluric lines.

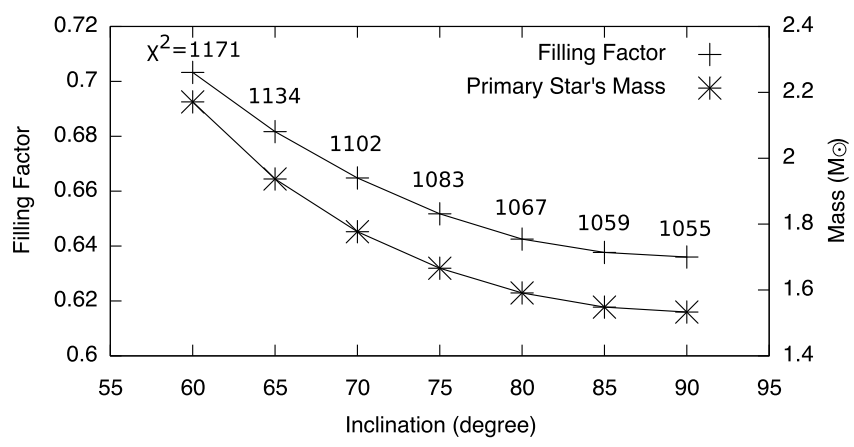

FIG. 3.- The above two curves show the best-fit filling factors of the secondary star and the inferred masses of the primary star versus the binary inclinations from $60^{\circ}$ to $90^{\circ}$ with the corresponding best-fit $\chi^{2}$ values.

of $\phi_{10}=0.5-1$ (i.e., $\phi_{20}=0.25-0.5$ in Figure 17a), during which the lowest radial velocity (RV) occurs at $\phi_{10}=0.75$ (i.e., $\left.\phi_{20}=0.375\right)$. Therefore, the radial velocity at $\phi_{10}=0.95$ (i.e., $\phi_{20}=0.47$ ) should be higher than that at $\phi_{10}=0.69$ (i.e., $\phi_{20}=0.34$ ). However, the result shows differently, indicating the invalidity of the irradiation case (see Figure 1 1a for a more clear demonstration). On the contrary, the observed radial velocities can be naturally explained in the case of ellipsoidal variation if the orbital phase zero is defined as the inferior conjunction (i.e., the companion is between the pulsar and the observer; Figure 1a).

\subsection{ELC fitting}

We used the Eclipsing Light Curve (ELC) code (Version 3; Orosz \& Hauschildt 2000) to model the optical lightcurves (i.e., $R$ - and $g$-bands) obtained from the MSU and Lulin observatories for a deeper understanding of the interacting binary. For the $R$-band data, we omitted the short $r$-band lightcurve (i.e., 2.4 hours) obtained from Lulin to prevent extra systematic uncertainties originated from the cross-calibrations between different filter systems (i.e., $r$ - and $R$-bands) and instruments. As ELC is capable of fitting RV, we also considered the two radial velocities to have a better constraint on the fitting result, despite the limited data quantity/quality. We also allowed a tiny phase shift between the phase-folded lightcurves and the models to further calibrate for the epoch of the inferior conjunction (i.e., the phase zero of the ELC models).

By (i) using the orbital period of $P_{\text {orb }}=20.8698 \mathrm{~h}$, (ii) assuming the effective temperature of the companion $T_{\text {eff }}=5750 \mathrm{~K}$ (it was not well determined because the ELC fit is insensitive to the companion temperature as ELC fits the normalized light curves) and the mass of the secondary star is $m_{2} \sim 0.4 M_{\odot}$ (by setting $\left.m_{2}=0.3-0.5 M_{\odot}\right)$, (iii) disabling the radiation heating effect, (iv) adopting the linear limb-darkening law van Hamme 1993) with a coefficient of $\kappa=0.6483$ (Sing 2010), and (v) setting a circular orbit (i.e. $e=0$ ), we fitted the lightcurves by varying four binary parameters, which are the binary inclination $(i)$, the mass ratio ( $q=m_{1} / m_{2}$, where $m_{1}$ is the pulsar mass), the orbital separation $(a)$, and the Roche-lobe filling factor $(\beta$, the ratio of the volume-averaged radii of the companion star and the Roche-lobe; Joss \& Rappaport 1984). With the built-in optimizer gridELC, we searched for the best-fit solution by minimizing the $\chi^{2}$ value and the least reduced chi-square of $\chi_{\nu}^{2}=3.1(\operatorname{dof}=344)$ was found at $i=90^{\circ}, q=6.8, a=4.6 R_{\odot}$, and $\beta=0.64\left(m_{1}=1.5 M_{\odot}\right.$ and $m_{2}=0.2 M_{\odot}$ are inferred). It is not a good fit statistically and the extreme inclination at the upper-bound may imply that the fit did not converge7. In addition, we found that the data can be fitted fairly well even if a fixed inclination angle of different value is used (see the similarity between the best-fit models at different inclinations in Figure 1 d). Therefore, instead of estimating the parameter uncertainties, we obtain and discuss the bestfit parameter sets at different inclinations from $i=90^{\circ}$ to $i=60^{\circ}$ with a step size of $5^{\circ}$. To elaborate the choice of $i=60^{\circ}$, it was chosen based on the $\chi^{2}$ values of the RVs on the ELC fits $\left(\chi_{\mathrm{RV}}^{2}\right)$. Despite the complexity of the optical emission revealed by the bad ELC fit, the RV

7 We once considered turning on the radiation heating effect to improve the fit. However, the flux overestimation in the valley at $\phi \sim 0.5$ is the main cause of the bad fitting. The radiation heating effect will even increase the predicted flux there to worsen the fit. 
data in principle would not be affected making $\chi_{\mathrm{RV}}^{2}$ a useful indicator to test the model validity. In this case, we chose a criteria of $\chi_{\mathrm{RV}}^{2}<3.84(95 \%$ c.l. for $d o f=1)$ to reject all other steps of $i<60^{\circ}$ with large $\chi_{\mathrm{RV}}^{2}$. We note that the selection heavily depends on the weighting on the RV data in the ELC fit (i.e., no weighting applied here) and therefore the rejection does not imply that the inclination has to be $i>60^{\circ}$. The selection simply indicates that the unweighted best-fit models with $i<60^{\circ}$ are inconsistent with the RV data and thus no discussion will be given on those fits.

Figure 3 shows the best-fit Roche-lobe filling factors and the inferred pulsar masses for $i=60^{\circ}-90^{\circ}$. As expected, the best-fit filling factor decreases with the inclination (from 0.70 to 0.64 ). All the best-fit results lead the primary star's mass to the range of $m_{1}=1.5-2.2 M_{\odot}$ (and $m_{2} \approx 0.2 M_{\odot}$ ), which is consistent with that of a pulsar. Certainly, the errors of the best-fit pulsar masses could be large (e.g., the uncertain companion mass as one of the major sources of error). Also, we found that the ELC fits are not robust. For instance, if we remove the constraints on the RV curve (i.e., the two Lijiang data points) and the companion mass (i.e., $\left.m_{2}=0.3-0.5 M_{\odot}\right)$, the best-fit solution of $i=90^{\circ}$ changes to $q=7.7$, $a=5.0 R_{\odot}$, and $\beta=0.63$, with which $m_{1}=2.0 M_{\odot}$ and $m_{2}=0.3 M_{\odot}$ are inferred (cf. Figure 3). Even two RV data points and a weak constraint on the companion mass are sufficient to significantly affect the fitting result. Therefore, we conclude that the best-fit parameters and the inferred masses are merely indicative. Detailed modelling (e.g., by adding hot/cool spots on the companion) as well as high-quality photometry sets and spectroscopic data of a complete orbit are required to place a further constraint on the pulsar mass. More imaging and spectroscopic observations are being planned to probe the system in the near-future.

\subsection{Possible X-ray Orbital Modulation}

As mentioned in 93 there is a significant variability seen in both Swift/XRT and Chandra data, which is possibly induced by the X-ray orbital modulation. We thus folded the lightcurve with the $20.87 \mathrm{~h}$ timing solution after converting the Chandra X-ray flux into the Swift/XRT band (i.e., $0.3-10 \mathrm{keV}$ ) and performing a barycentric correction to the data. Although the folded X-ray lightcurve does not cover a full orbital cycle, the X-ray variation is likely periodic with an X-ray minimum around the inferior conjunction (Figure 10). A similar phenomenon has been previously seen in the RB PSR J1023+0038 (Bogdanov et al. 2011; Tendulkar et al. 2014; Li et al. 2014). From the Chandra data bins, the X-ray maximum occurs around the superior conjunction (i.e., $\phi_{20} \sim 0.5$; observer-pulsarcompanion), although the Swift data favours the flux maximum around $\phi_{20}>0.5$.

\section{DISCUSSION AND CONCLUSION}

We presented a multi-wavelength study of 3FGL J0212.1+5320 and found that a RB MSP binary as its physical nature can naturally explain the entire data set. The $\mathrm{X} / \gamma$-ray spectral properties and the hourlytimescale orbital period are very similar to that of many known RBs (Table 1), revealing the first hint of 3FGL J0212.1+5320 as a RB candidate. The inferred primary star's masses from the best-fit ELC models are $1.5-$ $2.2 M_{\odot}$ that are consistent with that of a neutron star, though they are only indicative estimates. An hourly variability is seen in the Swift/Chandra joint lightcurve and it could be an orbital modulation, however, uncertainly. If the modulation is genuine, it could be caused by an intrabinary shock emission, through Doppler boosting with a pulsar-wrapping shock geometry (Li et al.]2014) or partial occultation by the companion (Bogdanov et al. 2011). All the observational evidence is pointing to the conclusion of 3FGL J0212.1+5320 as a newly-discovered RB system.

A bright optical counterpart (could be one of the brightest known for RBs) has been identified with a clear orbital modulation at 20.87 hours. We do not see an obvious non-uniform radiation heating to contribute to the orbital modulation and therefore the companion is probably not completely tidally locked. This may imply 3FGL J0212.1+5320 as a very young MSP system. According to Zahn (1977), the synchronization timescale of such a close binary is approximately $t_{\text {sync }} \sim 10^{4}\left(\left(1+q_{i}\right) / 2\right)^{2}\left(P_{i} / 1 \text { day }\right)^{4}$ years (equation 6.1 of Zahn 1977), where $q_{i}$ and $P_{i}$ are the initial mass ratio and orbital period, respectively 8 . Assuming an initial mass ratio of $q_{i}=2.8$ (i.e., $m_{1, i}=1.4 M_{\odot}$ and $\left.m_{2, i}=0.5 M_{\odot}\right)^{9}, P_{i} \approx 13 \mathrm{~d}$ gives $t_{\text {sync }} \gtrsim 10^{9}$ years and $P_{i} \approx 4 \mathrm{~d}$ gives $t_{\text {sync }} \gtrsim 10^{7}$ years. We took the calculated timescales for 3FGL J0212.1+5320 as lower limits because the orbital widening by the ablation from the pulsar (Chen et al. 2013), that would extend the synchronization process, was not considered in Zahn's work. In the case of $t_{\text {sync }} \geq 10^{7}$ years, the initial orbital period is actually close to the estimated value of PSR J2129-0429 (i.e., $P_{i} \approx 2.5 \mathrm{~d}$; Bellm et al. 2016), which has a long orbital period of $P=15.2 \mathrm{~h}$, comparable to 3FGL J0212.1+5320's. Obviously, a young age of 3FGL J0212.1+5320 (i.e., in the order of $10 \mathrm{Myr}$ ) would be a self-consistent explanation for the data. In fact, $\sim 10$ Myr old MSPs are rare but not impossible. For example, PSR J1823-3021A, one of the youngest MSPs known, has a characteristic age of 25 Myr (Freire et al. 2011). Searching for the radio/X-/ $\gamma$-ray pulsations of 3FGL J0212.1+5320 and computing the characteristic age would be useful to investigate the speculation.

Despite no heating effect seen, it is still highly likely that the companion is uniformly irradiated by the $\mathrm{X} / \gamma$ rays from the pulsar, resulting in a higher surface temperature than a $\sim 0.4 M_{\odot}$ star should have. As the companion mass is no longer the only dominant factor to determine the surface temperature, the assumption of $m_{2} \sim 0.4 M_{\odot}$ (see 4.2 ) could be overestimated. Considering the fact that all the fitting results indicate a lighter $m_{2}, m_{2} \lesssim 0.4 M_{\odot}$ would be more reasonable.

As the companion has a temperature close to that of the Sun, it is convenient to use the solar $R$-band absolute magnitude (i.e., $R=4.42 \mathrm{mag}$; Binney \& Merrifield 1998) to infer the distance of 3FGL J0212.1+5320. From the ELC model fits, the size of the companion is about

8 The equation presented here is slightly different from the one in Zahn 1977 because of the different definitions of the mass ratios.

9 The initial masses are both poorly known due to the highly uncertain accretion and ablation processes, and thus the values are merely estimated within reasonable ranges. 
TABLE 1

$\mathrm{X} / \gamma$-RAY PROPERTIES OF SOME KNOWN RBS IN THE PULSAR STATE AND 3FGL J0212.1+5320

\begin{tabular}{|c|c|c|c|c|c|c|}
\hline Name & $\begin{array}{c}\text { Spectral Curvature } \\
(\gamma \text {-ray; } \sigma)\end{array}$ & $\begin{array}{c}\text { Variability } \\
(\gamma \text {-ray })\end{array}$ & $\begin{array}{c}F_{0.1-100 \mathrm{GeV}} \\
\left(10^{-11} \mathrm{erg} \mathrm{cm}^{-2} \mathrm{~s}^{-1}\right) \\
\end{array}$ & $\Gamma_{X}$ & $\begin{array}{c}F_{0.5-7 \mathrm{keV}} \\
\left(10^{-13} \mathrm{erg} \mathrm{cm}^{-2} \mathrm{~s}^{-1}\right) \\
\end{array}$ & $F_{X} / F_{\gamma}$ \\
\hline \multicolumn{7}{|c|}{ (Generic $\gamma$-ray emitting RBs $)$} \\
\hline PSR J2129-0429 & 3.7 & 60.3 & 1.1 & 1.3 & 0.11 & $0.10 \%$ \\
\hline PSR J2339-0533 & 8.7 & 40.1 & 3.0 & 1.4 & 1.4 & $0.48 \%$ \\
\hline PSR J1628-3205 & 5.5 & 50.5 & 1.2 & (no & X-ray detection) & $<1.1 \%$ \\
\hline PSR J1048+2339 & 2.5 & 49.7 & 0.7 & (no & X-ray detection) & $<1.9 \%$ \\
\hline \multicolumn{7}{|c|}{ (Prospective tMSP Candidates in the Pulsar State) } \\
\hline PSR J2215+5135 & 6.8 & 56.9 & 1.4 & 1.8 & 1.0 & $0.74 \%$ \\
\hline PSR J1723-2837 & 3.3 & 55.7 & 1.8 & 0.9 & 24 & $13 \%$ \\
\hline \multicolumn{7}{|c|}{ (Fermi-detected tMSPs in the Pulsar State) } \\
\hline PSR J1227-4853 & $\cdots$ & $\ldots$ & 0.4 & 1.2 & 4.6 & $13 \%$ \\
\hline PSR J1023+0038 & $\ldots$ & $\ldots$ & 0.1 & 0.9 & 4.7 & $37 \%$ \\
\hline \multicolumn{7}{|c|}{ (Our Target) } \\
\hline 3FGL J0212.1+5320 & 6.3 & 51.5 & 1.7 & 1.3 & 14 & $7.9 \%$ \\
\hline
\end{tabular}

References: 3FGL (Acero et al. 2015); Tam et al. (2010); Kong et al. (2012); Linares (2014); Hui et al. (2015a); Xing \& Wang (2015); Deneva et al. (2016)

a3FGL curvature index: significance of the fit improvement between power-law and either LogParabola or PLExpCutoff spectrum type.

b3FGL variability index: a value greater than 72.44 indicates there is a less than $1 \%$ chance of being a steady source.

$R_{c} \approx 1 R_{\odot}$. After a proper scaling, the inferred distance is about $d \approx 0.8 \mathrm{kpc}$ leading to an X-ray luminosity of $L_{X} \approx 10^{32} \mathrm{erg} \mathrm{s}^{-1}$, which is relatively high among the known X-ray RBs in the pulsar state (when radio pulsations can be detected and $L_{X} \sim 10^{31}-4 \times 10^{32} \mathrm{erg} \mathrm{s}^{-1}$; Linares 2014). Since a high X-ray luminosity (i.e., $L_{X} \gtrsim 10^{32} \mathrm{erg} \mathrm{s}^{-1}$ ) in the pulsar state is a common feature of all three known transitional MSPs (tMSPs; i.e., PSR J1023+0038, PSR J1227-4853, and M28I), it has been suggested by Linares (2014) that $L_{X} \gtrsim 10^{32}$ is possibly a consequence of a stronger interaction between the pulsar and the companion, and therefore the higher X-ray luminosity could be a signature of a $\mathrm{RB}$ binary developing a strong accretion for the transition. One possibility is that the companion of a pre-transition (to the LMXB state) system has a stronger wind (i.e, a stronger inflow to the pulsar; see Takata et al. 2014 and $\mathrm{Li}$ et al. 2014 for the interpretation of a varying stellar wind as the transition trigger for PSR J1023+0038), which powers a stronger intrabinary shock X-ray emission. Based on the X-ray luminosity, two bright systems, PSR J2215+5135 $\left(L_{X}=1.3 \times 10^{32} \mathrm{erg} \mathrm{s}^{-1}\right)$ and PSR J1723-2837 $\left(L_{X}=2.4 \times 10^{32} \mathrm{erg} \mathrm{s}^{-1}\right.$; see Table 1 for their $\gamma / \mathrm{X}$-ray properties), have been suggested by Linares (2014) to be potential targets for state transitions in the near future. 3FGL J0212.1+5320 could be the third member of the group. In addition, we also examined the X-ray-to- $\gamma$-ray flux ratios of some known RBs and found that the flux ratios of the tMSPs (i.e., $\gg 1 \%$ ) are significantly larger than that of the "normal" RBs (i.e., $\lesssim 1 \%$ ). 3FGL J0212.1+5320 has a ratio of $7.9 \%$ that is consistent with the tMSP ones. One of the two prospective tMSP candidates, PSR J1723-2837, also has a large ratio of $13 \%$ (Table 1).

Certainly, the speculation is not mature and should not be taken conclusively. However, it is still worth paying attention to the X-ray activity of 3FGL J0212.1+5320 for any future transition. Even if it is not exhibiting any transition in the near future, 3FGL J0212.1+5320 could be one of the brightest RBs in X-rays and certainly is one of the best sources for studying the X-ray emissions of RBs.

No previous attempt of radio pulsation blind search for 3FGL J0212.1+5320 has been found in the literature (Ransom et al. 2011; Guillemot et al. 2012; Camilo et al. 2015). In fact, the system is likely radio-faint as no radio counterpart can be found in the $1.4 \mathrm{GHz}$ NRAO/VLA Sky Survey (NVSS), of which the detection limit is $~$ $2.5 \mathrm{mJy}$ Condon et al. 1998; Note: most of the radio MSPs found by targeting Fermi-LAT sources have flux densities much lower than $2.5 \mathrm{mJy}$ at $1.4 \mathrm{GHz}$; Ray et al. 2012). Nevertheless, a GBT observation is being planned for searching for radio coherent pulsations. Hopefully, this extreme RB MSP (i.e., high X-ray luminosity, bright optical companion, long orbital period, and potentially young age) can be confirmed soon.

After the submission of this paper, we became aware of a similar work by Linares et al. (2016), in which results including the measured orbital period, the radial velocity curve of the companion, the Chandra spectral analysis, and the redback MSP nature interpretation are consistent with ours. In particular, they have sampled a much better radial velocity curve, which would be very helpful in searching the radio $/ \gamma$-ray pulsations in the future.

Support for this work was partially provided by the National Aeronautics and Space Administration through Chandra Award Number DD5-16078X issued by the Chandra X-ray Observatory Center, which is operated by the Smithsonian Astrophysical Observatory for and on behalf of the National Aeronautics Space Administration under contract NAS8-03060. Support from NASA grant NNX15AU83G is gratefully acknowledged. AKHK and XH are supported by the Ministry of Science and Technology of Taiwan through grant 103-2628-M-007-003MY3 and 104-2811-M-007-059. XH is also supported by National Natural Science Foundation of China through grant 11503078. Jirong Mao is supported by the Hundred Talent Program of Chinese Academy of Sciences, the Key Research Program of Chinese Academy of Sciences (grant No. KJZD-EW-M06), and the Introducing Oversea Talent Plan of Yunnan Province. J.S. acknowledges support from a Packard Fellowship. The Lulin Observa- 
tory is operated by the Graduate Institute of Astronomy in National Central University, Taiwan. We acknowledge the support of the staff of the Lijiang $2.4 \mathrm{~m}$ telescope. Funding for the telescope has been provided by Chinese Academy of Sciences and the People's Government of Yunnan Province. The scientific results reported in this article are based in part on data obtained from the Chandra Data Archive. We acknowledge the use of public data from the Swift data archive. This publication makes use of data products from the Wide-field Infrared Survey Explorer, which is a joint project of the University of California, Los Angeles, and the Jet Propulsion Laboratory/California Institute of Technology, funded by the National Aeronautics and Space Administration.

\section{REFERENCES}

Abdo, A. A., Ackermann, M., Ajello, M., et al. 2010, ApJS, 188, 405

Abdo, A. A., Ajello, M., Allafort, A., et al. 2013, ApJS, 208, 17

Acero, F., Ackermann, M., Ajello, M., et al. 2015, ApJS, 218, 23

Alpar, M. A., Cheng, A. F., Ruderman, M. A., \& Shaham, J. 1982, Nature, 300, 728

Archibald, A. M., Stairs, I. H., Ransom, S. M., et al. 2009, Science, 324, 1411

Bellm, E. C., Kaplan, D. L., Breton, R. P., et al. 2016, ApJ, 816, 74

Binney, J., \& Merrifield, M. 1998, Galactic Astronomy

Bogdanov, S., Archibald, A. M., Hessels, J. W. T., et al. 2011, ApJ, 742, 97

Camilo, F., Kerr, M., Ray, P. S., et al. 2015, ApJ, 810, 85

Camilo, F., Reynolds, J. E., Ransom, S. M., et al. 2016, ApJ, 820 , 6

Cardelli, J. A., Clayton, G. C., \& Mathis, J. S. 1989, ApJ, 345, 245

Chen, H.-L., Chen, X., Tauris, T. M., \& Han, Z. 2013, ApJ, 775, 27

Cheng, K. S., Ho, C., \& Ruderman, M. 1986, ApJ, 300, 500

Condon, J. J., Cotton, W. D., Greisen, E. W., et al. 1998, AJ, 115,1693

Deneva, J. S., Ray, P. S., Camilo, F., et al. 2016, ApJ, 823, 105

Evans, P. A., Osborne, J. P., Beardmore, A. P., et al. 2014, ApJS, 210,8

Fan, Y.-F., Bai, J.-M., Zhang, J.-J., et al. 2015, Research in Astronomy and Astrophysics, 15, 918

Freire, P. C. C., Abdo, A. A., Ajello, M., et al. 2011, Science, 334 1107

Guillemot, L., Freire, P. C. C., Cognard, I., et al. 2012, MNRAS, 422,1294

Hui, C. Y., Hu, C. P., Park, S. M., et al. 2015a, ApJ, 801, L27

Hui, C. Y., Park, S. M., Hu, C. P., et al. 2015b, ApJ, 809, 68

Joss, P. C., \& Rappaport, S. A. 1984, ARA\&A, 22, 537

Kalberla, P. M. W., Burton, W. B., Hartmann, D., et al. 2005, A\&A, 440, 775

Kong, A. K. H., Huang, R. H. H., Cheng, K. S., et al. 2012, ApJ, 747, L3

Kong, A. K. H., Jin, R., Yen, T.-C., et al. 2014, ApJ, 794, L22

Li, K. L., Kong, A. K. H., Takata, J., et al. 2014, ApJ, 797, 111

Linares, M. 2014, ApJ, 795, 72

Linares, M., Miles-Páez, P., Rodríguez-Gil, P., et al. 2016, (MNRAS Submitted)
Mirabal, N., Charles, E., Ferrara, E. C., et al. 2016, ApJ, 825, 69 Monet, D. G., Levine, S. E., Canzian, B., et al. 2003, AJ, 125, 984 Munari, U., Sordo, R., Castelli, F., \& Zwitter, T. 2005, A\&A, 442,1127

Muslimov, A. G., \& Harding, A. K. 2003, ApJ, 588, 430

Oke, J. B. 1990, AJ, 99, 1621

Orosz, J. A., \& Hauschildt, P. H. 2000, A\&A, 364, 265

Papitto, A., \& Torres, D. F. 2015, ApJ, 807, 33

Papitto, A., Ferrigno, C., Bozzo, E., et al. 2013, Nature, 501, 517

Patruno, A., Archibald, A. M., Hessels, J. W. T., et al. 2014, ApJ, 781, L3

Pletsch, H. J., \& Clark, C. J. 2015, ApJ, 807, 18

Pletsch, H. J., Guillemot, L., Fehrmann, H., et al. 2012, Science, 338,1314

Ransom, S. M., Ray, P. S., Camilo, F., et al. 2011, ApJ, 727, L16

Ray, P. S., Abdo, A. A., Parent, D., et al. 2012, 2011 Fermi Symposium proceedings - eConf C110509

Roy, J., Ray, P. S., Bhattacharyya, B., et al. 2015, ApJ, 800, L12

Ruderman, M. A., \& Sutherland, P. G. 1975, ApJ, 196, 51

Saz Parkinson, P. M., Xu, H., Yu, P. L. H., et al. 2016, ApJ, 820, 8

Schlafly, E. F., \& Finkbeiner, D. P. 2011, ApJ, 737, 103

Sing, D. K. 2010, A\&A, 510, A21

Skrutskie, M. F., Cutri, R. M., Stiening, R., et al. 2006, AJ, 131, 1163

Strader, J., Chomiuk, L., Cheung, C. C., et al. 2015, ApJ, 804, L12

Stroh, M. C., \& Falcone, A. D. 2013, ApJS, 207, 28

Takata, J., Li, K. L., Leung, G. C. K., et al. 2014, ApJ, 785, 131

Tam, P. H. T., Hui, C. Y., Huang, R. H. H., et al. 2010, ApJ, 724, L207

Tauris, T. M. 2011, in Astronomical Society of the Pacific Conference Series, Vol. 447, Evolution of Compact Binaries, ed. L. Schmidtobreick, M. R. Schreiber, \& C. Tappert, 285

Tendulkar, S. P., Yang, C., An, H., et al. 2014, ApJ, 791, 77

van den Heuvel, E. P. J., \& van Paradijs, J. 1988, Nature, 334, 227

van Hamme, W. 1993, AJ, 106, 2096

Wright, E. L., Eisenhardt, P. R. M., Mainzer, A. K., et al. 2010, AJ, 140, 1868

Xing, Y., \& Wang, Z. 2015, ApJ, 808, 17

Zacharias, N., Finch, C. T., Girard, T. M., et al. 2013, AJ, 145, 44

Zahn, J.-P. 1977, A\&A, 57, 383 